\title{
Influence of MSI and I8q LOH markers on capecitabine adjuvant monotherapy in colon cancer patients
}

This article was published in the following Dove Press journal: Pharmacogenomics and Personalized Medicine

\author{
Nadica Matevska- \\ Geshkovska' \\ Marija Staninova-Stojovska' \\ Aleksandra Kapedanovska- \\ Nestorovska' \\ Natalija Petrushevska- \\ Angelovska ${ }^{2}$ \\ Milco Panovski ${ }^{3}$ \\ Biljana Grozdanovska² \\ Nenad Mitreski² \\ Aleksandar Dimovski' \\ 'Center for Biomolecular \\ Pharmaceutical Analyses, Faculty of \\ Pharmacy, Ss. Cyril and Methodius \\ University in Skopje, Skopje, \\ Macedonia; ${ }^{2}$ University Clinic for \\ Oncology and Radiotherapy, Ss. Cyril \\ and Methodius University in Skopje, \\ Skopje, Macedonia; ${ }^{3}$ University Clinic \\ for Abdominal Surgery, Ss. Cyril \\ and Methodius University in Skopje, \\ Skopje, Macedonia
}

Purpose: The aim of this study was to evaluate whether pretreatment analysis of selected molecular markers can be used for the prediction of disease-free survival (DFS)/overall survival (OS) of capecitabine adjuvant monotherapy in colon cancer patients.

Patients and methods: A total of 126 patients enrolled in a capecitabine Phase IV clinical trial were analyzed for microsatellite instability (MSI), 18q loss of heterozygosity (LOH), thymidylate synthase (TYMS) 5' variable number of tandem repeat (VNTR), and methylene tetrahydrofolate reductase (MTHFR) C677T variants. The significance in predicting 5-year DFS/OS was assessed by Kaplan-Meier and Cox regression analyses.

Results: The MSI-high (MSI-H) genotype was significantly associated with DFS (HR 0.205, 95\% CI $0.05-0.88, P=0.033$ ) and OS (HR 0.208, 95\% CI 0.05-0.89, $P=0.035$ ) compared to the microsatellite stable genotype. In models stratified according to clinicopathologic characteristics, the MSI-H genotype remained a positive predictive factor for DFS/OS only in patients with stage III $(P=0.023)$ and patients with tumors localized proximally to the splenic flexure $(P=0.004)$. Distal colon cancers with $18 \mathrm{q}$ LOH have a greater survival rate when treated with capecitabine than patients with stable tumors $(81.3 \%$ vs $50.0 \%$, HR for relapse $0.348,95 \%$ CI $0.13-0.97$, $P=0.043$ ). TYMS 5 'VNTR and MTHFR C677T variants were not associated with DFS or OS. Conclusion: MSI and 18q LOH markers have the potential to be utilized in the selection of colon cancer patients eligible for capecitabine adjuvant monotherapy.

Keywords: gastrointestinal cancer, microsatellite instability, $18 \mathrm{q}$ allelic imbalance, prognostic marker

\section{Introduction}

Colorectal cancer (CRC) is the third most common cancer in men (746,000 cases, $10.0 \%$ of the total) and the second in women $(614,000$ cases, $9.2 \%$ of the total) worldwide. ${ }^{1,2}$ Over the past decade, CRC mortality rates have declined in many longstanding economically developed countries as a consequence of improved screening programs and the availability of newer and more effective agents for adjuvant and palliative chemotherapies. On the other hand, this is not the case in low-resource countries of Central and Eastern Europe where CRC mortality rates continue to increase. ${ }^{1,2}$

The current standard primary treatment of CRC is complete surgical resection of the tumor followed by systemic chemotherapy in patients with a high risk of recurrence. 5-Fluorouracil (5-FU) in combination with leucovorin (LV), with or without oxaliplatin (FOLFOX), is the most widely used treatment for an adjuvant setting of CRC. ${ }^{3}$ All of these agents are administered intravenously (by bolus or infusion), thereby causing
Correspondence: Aleksandar Dimovski Center for Biomolecular Pharmaceutical Analyses, Faculty of Pharmacy, Ss. Cyril and Methodius University in Skopje, Majka Tereza 47, 1000 Skopje, Macedonia Tel +38923 II 9694 ext 109 Fax +38923 I2 3054 Email adimovski@ff.ukim.edu.mk 
significant inconvenience to patients. Xeloda ${ }^{\circledR}$ (capecitabine; Hoffmann-La Roche Ltd., Basel, Switzerland), an oral fluoropyrimidine, is a prodrug of 5-FU. Phase III clinical trials demonstrated that capecitabine is at least as effective as bolus 5 -FU/LV in terms of time to disease progression and overall survival (OS), but achieves significantly higher response rates, has improved tolerability with significantly lower incidence of stomatitis, nausea, and alopecia, and has the advantage of oral administration. ${ }^{4,5}$ Furthermore, some pharmacoeconomic analyses have highlighted that capecitabine is more cost-effective when compared with 5 -FU/LV. ${ }^{6,7}$ Most cost savings are attributable to reduced administration costs and lower incidence of adverse effects, that is, hospitalization for treatment-related adverse events. The only clinical adverse event seen more commonly with capecitabine is the hand-foot syndrome, which, however, is never life threatening. ${ }^{4}$

Interindividual differences in toxicity and response to chemotherapy are currently observed in practically all available $\mathrm{CRC}$ treatment regimens. Thus, to improve the outcome of the treatment, efforts must be directed toward the identification of patients who are likely to respond to a specific therapy, those who will experience severe toxicities, and those who will benefit from chemotherapy in the adjuvant setting. Stage II CRC patients present with no lymph node or distant metastases and therefore have a better overall prognosis. Surgical resection is highly effective for a localized disease, but nevertheless, a significant portion of these patients develop recurrence. Currently, these high-risk patients are identified by tumors that not only penetrate the bowel wall but also show evidence of adhesion to or invasion of the surrounding structures, free perforation, or obstruction. ${ }^{8}$ Clearly, there is a need for novel predictive factors, which will be used together with nodal involvement in the recognition of stage II patients who are likely to experience relapse and therefore, in need of adjuvant treatment. Most of the studies evaluating molecular markers for the prediction of efficacy of the adjuvant therapy in CRC evaluate patients receiving either 5-FU/LV monotherapy or a combination of 5-FU/LV with irinotecan or oxaliplatin. However, there are very scarce data on capecitabine-based protocols. Identifying the subset of patients who will most probably respond to capecitabine monotherapy is imperative since oral administration is preferred by patients, is more cost-effective, and has less severe adverse events and inconveniences attributable to combinational therapies.

The main aim of this study was to evaluate whether selected molecular parameters can be used as predictive markers for disease-free survival (DFS) and OS in colon cancer patients treated with capecitabine adjuvant monotherapy.
Molecular markers that were hypothesized as predictive for the efficacy of the therapy were microsatellite instability (MSI), allelic imbalance at the 18q chromosomal arm (demarcated as a loss of heterozygosity $[\mathrm{LOH}]-18 \mathrm{q} \mathrm{LOH})$, and thymidylate synthase (TYMS) $5^{\prime}$ variable number of tandem repeat (VNTR) and methylene tetrahydrofolate reductase (MTHFR) C677T variants.

\section{Materials and methods Study design}

The study was designed as a local observational open-label trial (EU PAS register number ML20122). A total of 126 patients with histologically proven stage III or high-risk stage II colon cancer were recruited between January 1, 2006 and December 31, 2009, at the University Clinic for Oncology and Radiotherapy, Skopje, Macedonia. Patients with rectal cancer, previous malignancies, previous chemotherapy or immunotherapy, moderate or severe renal impairment, clinically significant cardiovascular disease, central nervous system disorders, chronic inflammatory bowel disease, bowel obstruction or active peptic ulcer, and patients with known dihydropyrimidine dehydrogenase polymorphisms were not included in the study. The study was approved by the ethical review board of the Faculty of Medicine, Skopje (03-525; February 16, 2006) and written informed consent and a questionnaire that contained the most relevant data on family history were obtained from each patient. All recruited patients underwent surgical resection of the tumors and were treated with capecitabine adjuvant monotherapy according to the following protocol: capecitabine $1,250 \mathrm{mg} / \mathrm{m}^{2}$, twice daily 12 hours apart (total daily dose $2,500 \mathrm{mg} / \mathrm{m}^{2}$ ) for 14 days. The treatment was repeated every 3 weeks for a total of eight cycles ( 24 weeks). A total of 119 $(94.44 \%)$ patients completed the treatment protocol. The treatment was stopped in seven patients due to severe adverse events (diarrhea, $n=1)$ or patient choice or frailty $(n=6)$. After completion of the therapy, follow-up visits were scheduled every 3 months in the first year, every 6 months in the next 2 years, and once a year in the following 2 years until a total of 60 months since the completion of chemotherapy. According to the Schedule of Assessments, each follow-up visit included a blood hematology test (complete blood count), blood chemistry tests (serum creatinine, urea and electrolytes, total bilirubin, aspartate aminotransferase, alanine aminotransferase, alkaline phosphatase, total protein, and albumin), tumor marker tests (CEA and CA 19-9), and abdominal ultrasound. The final evaluation of each patient was done at the end of the trial, 60 months since the completion of treatment, or earlier in case of premature withdrawal. 


\section{$\mathrm{MSI}$ and $\mathrm{I} 8 \mathrm{q} \mathrm{LOH}$ analysis}

Paired peripheral blood (EDTA) and formalin-fixed, paraffinembedded (FFPE) tumor tissue samples were obtained from each patient. DNA from both samples was extracted using the QIAamp DNA Blood Mini Kit and QIAamp DNA FFPE Tissue Kit (Qiagen NV, Venlo, the Netherlands) according to the manufacturer's protocols. MSI and $18 \mathrm{q} \mathrm{LOH}$ were analyzed with multiplex fluorescent PCR followed by capillary electrophoresis on 310 or 3500 Automated Genetic Analyzers (Thermo Fisher Scientific, Waltham, MA, USA). The MSI status was determined using 10 short tandem repeat markers: BAT25, BAT26, D2S123, D5S107, D5S346, D17S250, D18S46, D18S58, D18S61, and D18S535 derived from the panel of microsatellite loci defined by the National Cancer Institute. ${ }^{9}$ The tumor samples were classified as MSI-high (MSI-H) if instability was present at $>30 \%$ of the loci screened, MSI-low (MSI-L) if at least one but $<30 \%$ of the loci showed instability, or microsatellite stable (MSS) if all loci were stable. For statistical analysis, MSI-L and MSS patients were combined in one group since tumors with low-frequency MSI are not biologically distinct from those exhibiting microsatellite stability. ${ }^{10}$ Four markers were used for the 18q LOH analysis: D18S46, D18S58, D18S61, and D18S535. 18q LOH was scored only in MSS tumors and was defined as the peak ratio of tumor to normal $>1.35$ or $<0.65$ for each marker. Markers with monoallelic results or MSI were determined to be noninformative. Positivity for $18 \mathrm{q}$ LOH was defined if at least one of the four markers showed the presence of LOH. Negativity for 18q LOH was defined as the presence of at least two informative markers with the absence of $\mathrm{LOH}$.

\section{MTHFR C677T genotyping}

MTHFR C677T polymorphism (rs 1801133) was genotyped by allele discrimination PCR on a Stratagene Mx3005P (Agilent Technologies, Santa Clara, CA, USA) real-time PCR system using TaqMan ${ }^{\circledR}$ SNP genotyping assay (reference C__1202883_20; Thermo Fisher Scientific). The genotypes were determined in a reaction mix containing $20 \mathrm{ng}$ DNA in a total volume of $25 \mu \mathrm{L}$ according to the manufacturer's recommended protocol. Positive and negative controls were included on each plate and reproducibility was checked by regenotyping $10 \%$ of the cases.

\section{TYMS 5'VNTR analysis}

TYMS 5'VNTR variants were analyzed using a custom designed assay. The 5'-TSER of TYMS gene was amplified using the following primers: 5'GTG-
GCTCCTGCGTTTCCCCC3' (forward primer) and 6-FAM5'GCTCCGAGCCGGACACAGGCA3' (6-FAM labeled reverse primer). A total of $100 \mathrm{ng}$ of DNA was used in 25 $\mu \mathrm{L}$ final volume including $1 \times$ Hot Start Buffer B2, $1 \times$ solution $\mathrm{S}, 2 \mathrm{mM} \mathrm{Mg}{ }^{2+}, 0.2 \mathrm{mM}$ of each dNTP, $1 \mu \mathrm{M}$ of both primers, and $1 \mathrm{U}$ of HOT FIREPol ${ }^{\circledR}$ DNA Polymerase (Solis BioDyne, Tartu, Estonia). PCR was performed on Veriti (Thermo Fisher Scientific) using a touchdown program: initial denaturation at $95^{\circ} \mathrm{C}$ for 12 minutes; 10 cycles of 1 minute at $95^{\circ} \mathrm{C}, 1.5$ minutes at $75^{\circ} \mathrm{C}$ with $1{ }^{\circ} \mathrm{C}$ reduction per cycle; 25 cycles of 1 minute at $95^{\circ} \mathrm{C}$ and 1.5 minutes at $65^{\circ} \mathrm{C}$; and final elongation at $72^{\circ} \mathrm{C}$ for 10 minutes. Five microliters of the PCR product was digested with the restriction enzyme HaeIII according to the manufacturer's instructions (New England Biolabs, Ipswich, MA, USA). The digest is used to discriminate the $3 R C$ allele from the $3 R G$ allele since $G>C$ SNP in the $5^{\prime}$-TSER 3RC allele affects the HaeIII site. PCR and the digested product were combined in a 1:5 ratio and detected by capillary electrophoresis on a 3500 Automated Genetic Analyzer (Thermo Fisher Scientific). The results were reported as high expression alleles $(2 \mathrm{R} / 3 \mathrm{G}, 3 \mathrm{C} / 3 \mathrm{G}$, and $3 \mathrm{G} / 3 \mathrm{G}$ genotypes) and low expression alleles $(2 \mathrm{R} / 2 \mathrm{R}$, $2 \mathrm{R} / 3 \mathrm{C}$, and $3 \mathrm{C} / 3 \mathrm{C}$ genotypes).

\section{Statistical analysis}

Descriptive data were tabulated as mean \pm standard deviation and range for continuous variables and number (and percentage) for categorical variables. Differences in demographic and clinical characteristics of patients were tested using the unpaired Student's $t$-test (continuous variables) or Fisher's exact test (categorical variables). The primary end point of the study was DFS, defined as the time from the end of the adjuvant therapy to the first recurrence. OS, which was defined as the time from the end of the adjuvant therapy to colon cancer-specific death, was the secondary end point. In detail, relapse-free patients in this study were: 1) those who were alive and without recurrences at the end of the followup (5 years after therapy, $\mathrm{N}=45$ ); 2 ) those who died of causes not related to colon cancer and without recurrences during follow-up (the end of follow-up is the date of death, $N=4$ ); 3 ) those who were alive and without recurrences, but because of personal reasons decided not to adhere to regular followup visits (the end of follow-up is the last follow-up control, $\mathrm{N}=24, \mathrm{DFS}_{\text {avg }}=45$ months). Only patients who completed the treatment protocol and had follow-up data for at least 18 months were evaluated for DFS and OS $(n=110)$. Survival curves were generated according to the Kaplan-Meier method and univariate survival distributions were compared 
with the use of the log-rank test. HR and 95\% CI for univariate and multivariate models were computed with the use of Cox proportional hazards regression model. All statistical analyses were conducted using the IBM SPSS software v22.0 (IBM Corporation, Armonk, NY, USA).

\section{Results}

Disease relapse was observed in 38 patients $(34.55 \%)$. The mean DFS for the relapse group was 17.92 months (95\% CI 13.47-22.63) and for the relapse-free group 60.14 months (95\% CI 54.39-65.44). None of the clinicopathologic variables (gender, age, tumor localization, and stage) was predictive for DFS/OS in the study group (log-rank test for DFS $P$-values: $0.65,0.64,0.31$, and 0.42 , respectively; data not shown).

The general characteristics of the study participants and the association of the tested markers with the clinicopathologic variables are presented in Table 1. MSI was observed in 17 patients $(17.71 \%)$, whereas $18 \mathrm{q} \mathrm{LOH}$ was present in $44(55.70 \%)$ MSS patients. MSI-H was associated with localization of the tumors to a site proximal to the splenic flexure $(P=0.012)$ (Table 1$)$. In all other aspects, there was no statistical significance among patients exhibiting MSI-H and MSS, as well as between patients with $18 \mathrm{q} \mathrm{LOH}$ and 18 stable tumors (Table 1).

Significant interaction was observed between the MSI status and benefit of treatment. The rate of 5-year DFS among patients with tumors exhibiting MSI-H (88.2\%) was significantly higher than DFS in patients with MSS tumors $(60.8 \% ; P=0.032)$ (Table 2; Figure 1). In the multivariate analysis adjusted for age, gender, stage, and tumor localization, the MSI-H genotype was significantly associated with DFS (HR 0.205, 95\% CI 0.05-0.88, $P=0.033$ ) and OS (HR $0.208,95 \%$ CI $0.05-0.89, P=0.035)$ compared to the MSS genotype (Table 2). In models stratified according to clinicopathologic characteristics, the MSI-H genotype remained a positive predictive factor for DFS/OS only in patients with stage III tumors $(P=0.023)$ and patients with tumors localized proximally to the splenic flexure $(P=0.004$, Table 2 ; Figure 2$)$. None of the MSI-H patients with stage III or proximal tumors developed relapse.

In MSS patients, there was no significant difference in DFS (HR 0.618 , 95\% CI $0.30-1.29, P=0.199$ ) or OS (HR $0.628,95 \%$ CI $0.31-1.31, P=0.213$ ) according to the $18 \mathrm{q}$ LOH status (Table 3; Figure 3). We further examined the effect of $18 \mathrm{q}$ LOH in strata according to tumor stage, localization, gender, and age. Patient mortality did not significantly differ according to any clinicopathologic characteristic except for tumor localization. We found that patients with $18 \mathrm{q} \mathrm{LOH}$ distal colon cancer have a significantly higher survival rate than patients with chromosomal stable tumors $(81.3 \%$ vs $50.0 \%, P=0.027$, Figure 4$)$. Multivariate analyses on the effect of $18 \mathrm{q} \mathrm{LOH}$ in patients treated with capecitabine adjuvant monotherapy showed better survival only in patients with distal localization of their tumors (HR for relapse $0.348,95 \%$ CI $0.13-0.97, P=0.043)$, but not in patients with proximal

Table I Demographics and clinical characteristics of the study participants and the association of MSI and I8q LOH status with clinicopathologic variables

\begin{tabular}{|c|c|c|c|c|c|c|c|}
\hline & \multirow{2}{*}{$\begin{array}{l}\text { All patients } \\
(\mathrm{N}=|| 0)\end{array}$} & \multicolumn{3}{|l|}{ MSI status ${ }^{a}$} & \multicolumn{3}{|c|}{ I8q LOH status ${ }^{b}$} \\
\hline & & $\begin{array}{l}\text { Patients with } \\
\text { MSI-H tumors } \\
(\mathrm{N}=17)\end{array}$ & $\begin{array}{l}\text { Patients with } \\
\text { MSS tumors } \\
(\mathrm{N}=79)\end{array}$ & $P$-value & $\begin{array}{l}\text { Patients with } \\
\text { I8q LOH } \\
\text { tumors } \\
(\mathrm{N}=44)\end{array}$ & $\begin{array}{l}\text { Patients with } \\
18 q \text { intact } \\
\text { tumors } \\
(\mathrm{N}=34)\end{array}$ & $P$-value \\
\hline \multicolumn{8}{|l|}{ Gender } \\
\hline Male & $60(54.6)$ & $8(15.4)$ & $44(84.6)$ & $0.40 \mathrm{I}$ & $26(60.5)$ & $17(39.5)$ & 0.583 \\
\hline Female & $50(45.4)$ & $9(20.5)$ & $35(79.5)$ & & $18(5 \mid .4)$ & $17(48.6)$ & \\
\hline \multicolumn{8}{|l|}{ Age (years) } \\
\hline Mean $( \pm S D)$ & $59.5( \pm 9.2)$ & $59.3( \pm 10.3)$ & $59.7( \pm 8.4)$ & 0.998 & $59.1( \pm 9.2)$ & $60.8( \pm 7.1)$ & 0.814 \\
\hline Range & $36-81$ & $40-8 I$ & $40-74$ & & $40-7 \mid$ & $44-74$ & \\
\hline \multicolumn{8}{|c|}{ Tumor localization } \\
\hline Proximal & 42 (38.2) & II (29.7) & $26(70.3)$ & 0.012 & $12(46.2)$ & $14(53.8)$ & 0.258 \\
\hline Distal & $68(61.8)$ & $6(10.2)$ & $53(89.8)$ & & $32(61.5)$ & $20(38.5)$ & \\
\hline \multicolumn{8}{|l|}{ Tumor stage } \\
\hline II & $62(56.4)$ & $10(19.2)$ & $42(80.8)$ & 0.406 & $25(61.0)$ & $16(39.0)$ & 0.765 \\
\hline III & $48(43.6)$ & $7(15.9)$ & $37(84.1)$ & & $19(51.4)$ & $18(48.6)$ & \\
\hline
\end{tabular}

Notes: Data are presented as numbers with percentages in parentheses unless otherwise indicated. aissing data are due to the low quality of FFPE tumor specimens $(\mathrm{N}=14)$. ${ }^{b}$ Data are shown only for patients with MSS tumors and informative $18 \mathrm{q} \mathrm{LOH}(\mathrm{N}=78)$. Bold value indicates significance.

Abbreviations: FFPE, formalin-fixed, paraffin-embedded; LOH, loss of heterozygosity; MSI, microsatellite instability; MSS, microsatellite stable; MSI-H, MSI-high. 


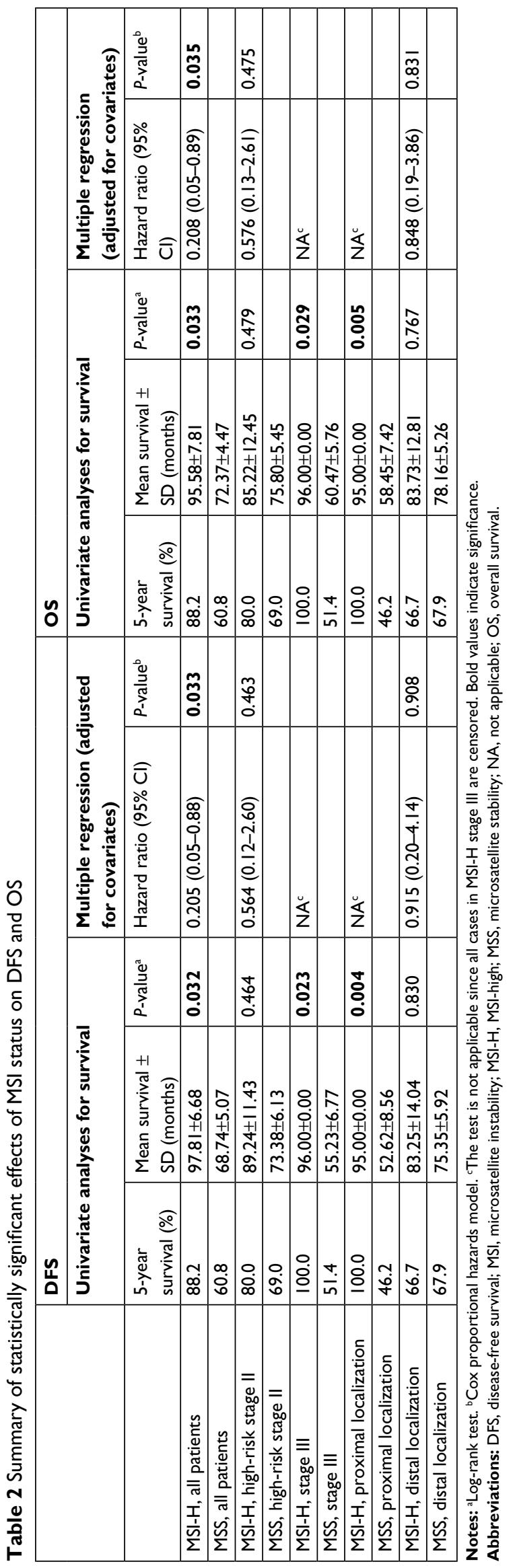

tumor localization (HR for relapse 1.140, 95\% CI 0.39-3.34, $P=0.812$ ) (Table 3).

TYMS 5'VNTR and MTHFR C677T variants were not associated with DFS (HR for TYMS high expression alleles 1.17 [0.61-2.24], $P=0.642$ and for MTHFR 677TT genotype 0.29 [0.04-2.13], $P=0.225$ ). When overall mortality was used as an end point, the results remained consistent. Furthermore, in models stratified according to clinicopathologic characteristics, TYMS and MTHFR variants did not show any significant differential effect on relapse or mortality in any of the evaluated strata.

\section{Discussion}

Colon cancer arises through at least two distinct pathways of genetic instability: one involving a chromosomal instability presenting as $\mathrm{LOH}$, and the other involving MSI. Patients with MSI-H tumors have distinct clinical and pathological features irrespective of the tumor origin and more favorable prognosis in terms of survival if treated with surgery alone. ${ }^{11-15}$ Regarding the relative benefits of chemotherapy among MSI-H and MSS patients, a current consensus has been reached regarding the lack of benefit of 5-FU-based therapies in patients with the MSI-H genotype. ${ }^{16}$ Several studies have shown that only patients with MSS tumors have significant survival benefit with the 5-FU-based treatment ${ }^{15,17-19}$ and that this treatment might even be harmful to patients harboring MSI-H tumors since 5-FU masks the overall good prognostic impact of the MSI-H genotype. ${ }^{20,21}$ However, the predictive utility of MSI still remains controversial given that some studies have failed to demonstrate the negative predictive impact of MSI, ${ }^{22-25}$ and some have suggested that MSI-H is strongly prognostic for DFS and OS and that patients with MSI-H tumors maintain their survival advantage in comparison with MSS patients in the presence of 5-FU. ${ }^{26-32}$ Correspondingly, our data showed that the MSI-H genotype is a positive predictive factor for DFS and OS in patients treated with capecitabine adjuvant monotherapy. The only two cases of MSI-H-positive patients with recurrence within the 5-year follow-up have similar clinicopathologic characteristics: age at diagnosis $>60$ years, adenocarcinoma with distal localization (sigmoid colon) and local invasion in the pericolorectal tissues (stage T4). In contrast, the relapse-free patients with T4 tumor stage have very different characteristics: age at diagnosis $<60$ years, BRAF-mutated mucinous adenocarcinoma located proximal to the splenic flexure. This suggests that the present tumor invasion in the pericolorectum cannot be a sole reason for the decreased benefit of the capecitabine monotherapy, but rather the recurrence is most likely influenced by different 
A

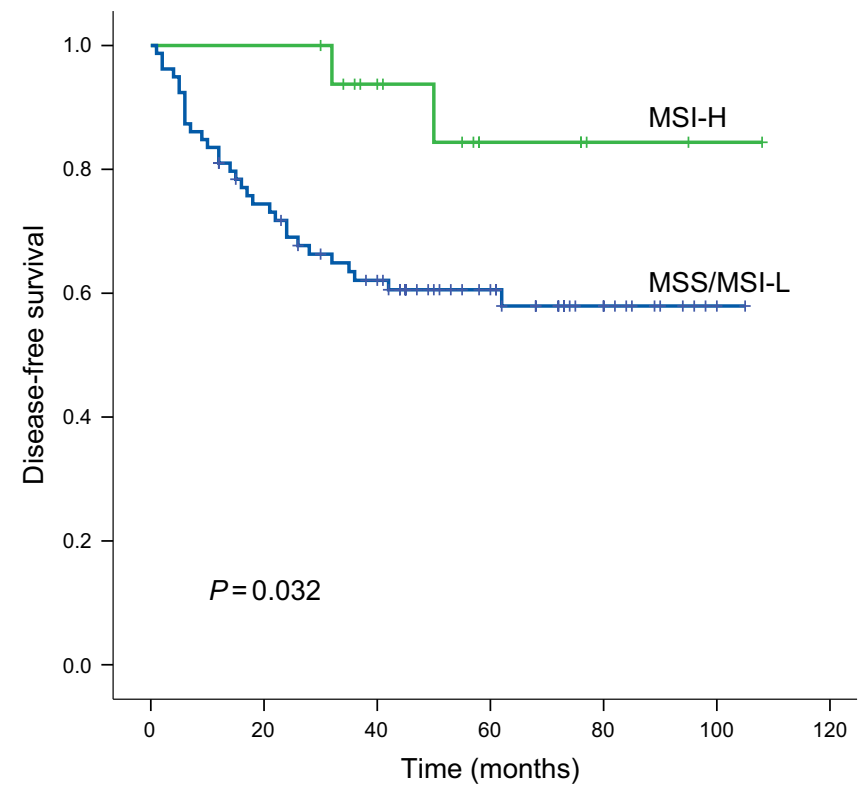

B

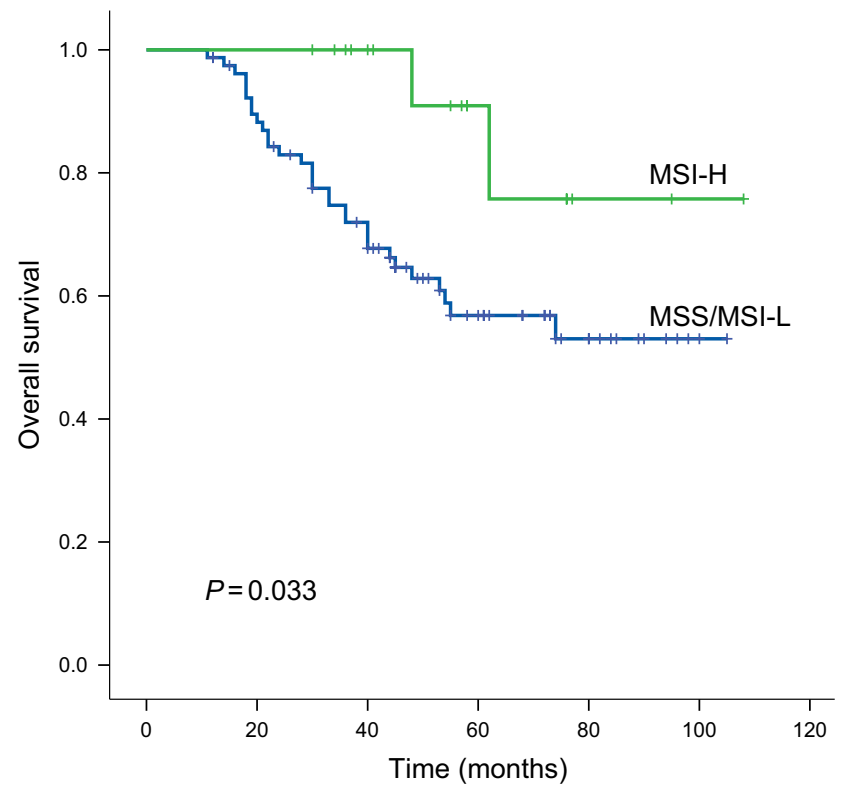

Figure I Kaplan-Meier estimates for disease-free survival (A) and overall survival (B) by MSI status in patients treated with capecitabine adjuvant monotherapy. Abbreviations: MSI, microsatellite instability; MSI-H, MSI-high; MSI-L, MSI-low; MSS, microsatellite stable.

molecular changes that arise due to different mechanisms of MSI occurrence. This is clinically important since it gives an opportunity for further stratification of patients with the MSI-H genotype. Patients with proximal MSI-H tumors that arise from serrated adenomas have a better outcome regardless of the stage of the disease and are candidates for capecitabine monotherapy. Thus, these patients would be spared of the unnecessary burden of combination chemotherapy, that is, treatment-related toxic effects and reduced quality of life. In contrast, the positive predictive effect of the MSI-H genotype is not apparent in patients with distally located tumors treated with capecitabine monotherapy and they should be considered for the standard FOLFOX protocol. However, although this is a prospective study and all patients received the same clinical management through the treatment courses, our findings need to be interpreted with caution since the small number of patients made the subgroup analysis difficult. For example, the most probable reason for stage III MSI-H patients showing better survival than high-risk stage II MSI-H patients is the enrichment of patients with proximal tumors who have better prognosis within the stage III group due to the small sample size. Furthermore, since we did not have a control group comprising patients who did not receive adjuvant chemotherapy, it remains unclear if this association is due to the treatment alone or to the combination with the preferable prognostic impact of the MSI-H genotype. Nonetheless, it is worth noting, based on our results, that the MSI-H genotype could not be associated with a reduced survival rate in patients treated with capecitabine, as reported by some research groups, ${ }^{20,21}$ and therefore, these patients should not be excluded from 5-FU-based adjuvant therapy.

Allelic loss at the $18 \mathrm{q}$ chromosomal arm occurs with a great frequency in the carcinogenic process of colon cancer. This region contains several important tumor suppressor genes, such as DCC, SMAD4 (DPC4), SMAD2, and CABLES1. ${ }^{33-35}$ Given its potential use as a prognostic marker, a number of studies have evaluated the association between 18q LOH and CRC outcome. However, the results are difficult to interpret since reports are almost evenly divided between those finding that $18 \mathrm{q} \mathrm{LOH}$ predicts poor survival $^{34-40}$ and those showing no association. ${ }^{41-46}$ One possible explanation for the inconsistency of the association is that many of the initial studies did not consider the potential confounding effect of MSI-H, which is typically associated with improved patient survival. Because chromosomal instability and MSI are almost mutually exclusive, ${ }^{47}$ the association between $18 \mathrm{q} \mathrm{LOH}$ and poor prognosis in these studies might be simply due to the enrichment of MSI-H tumors within the group of $18 \mathrm{q}$ stable patients. In contrast to the extensive exploration of the influence of the $18 \mathrm{q}$ LOH markers on the CRC patients' prognosis, there are still limited data regarding the effect of these markers on survival in patients receiving 5-FU-based adjuvant chemotherapy. Results from these studies generally demonstrate that the retention of the 
A

High risk stage II

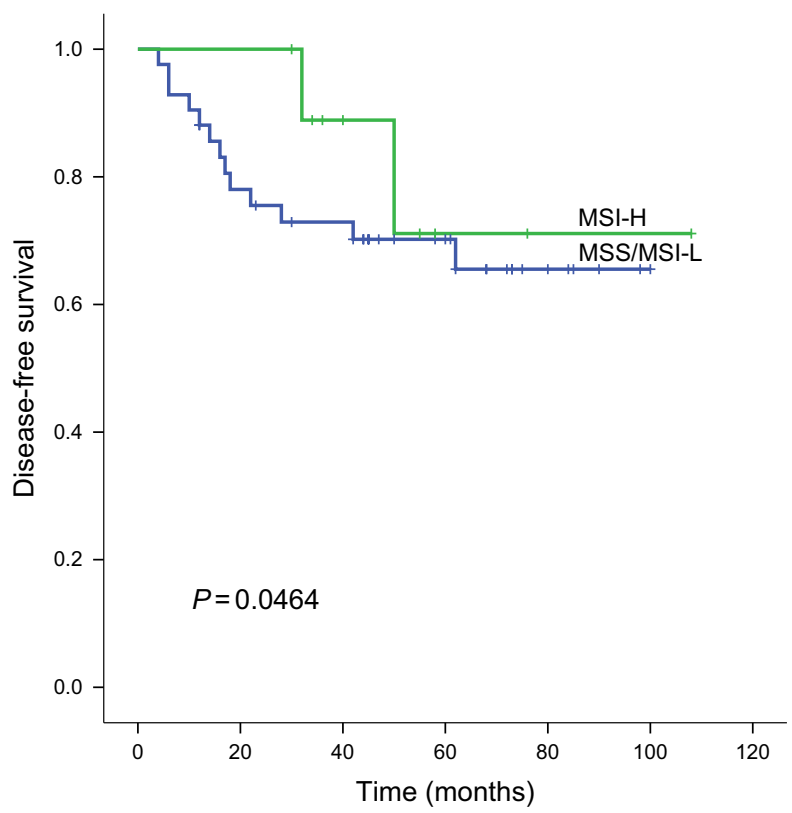

C

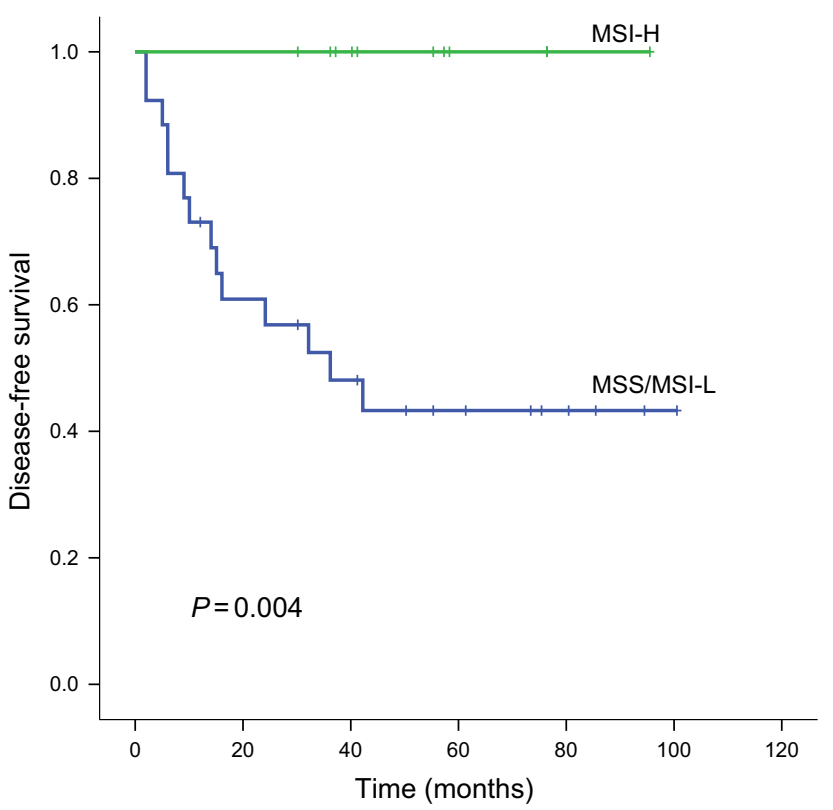

B

Stage III

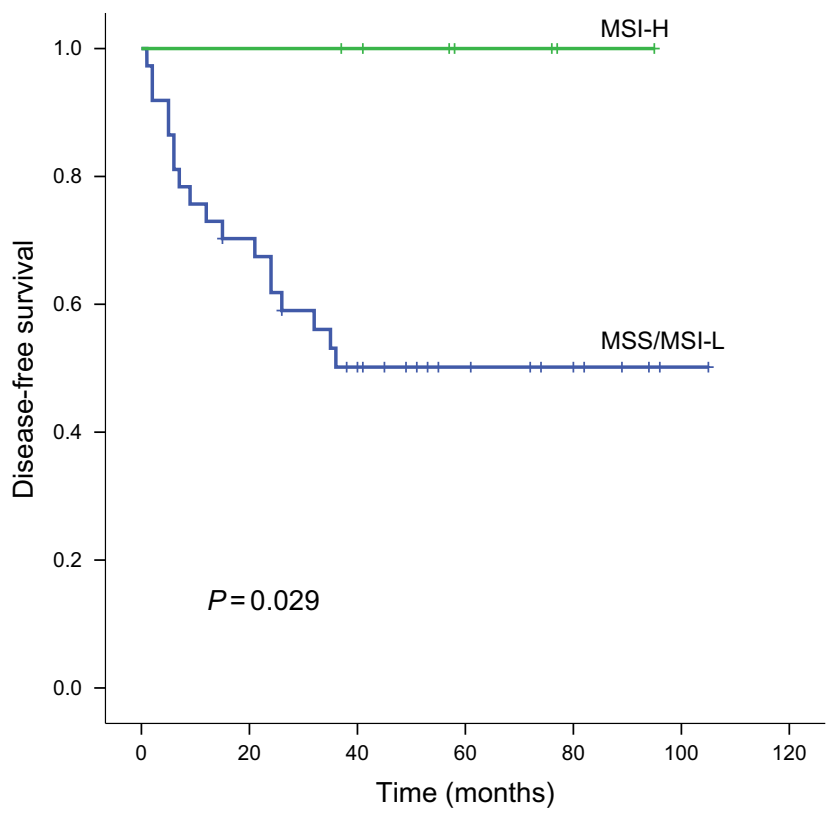

D

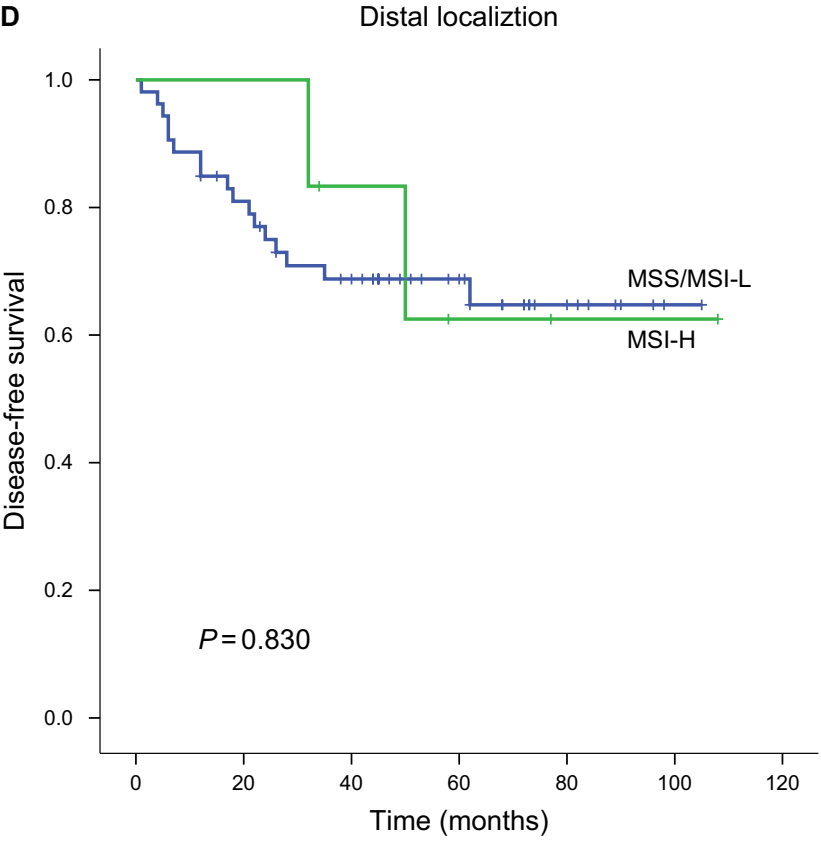

Figure 2 Kaplan-Meier curves of disease-free survival rates according to MSI status in CRC patients receiving capecitabine adjuvant monotherapy stratified according to the stage (A, B) and localization (C, D) of the tumors.

Abbreviations: MSI, microsatellite instability; MSI-H, MSI-high; MSI-L, MSI-low; MSS, microsatellite stable; CRC, colorectal cancer.

$18 \mathrm{q}$ alleles in MSS cancer points to a favorable outcome after adjuvant chemotherapy in colon cancer patients. . $^{35,48}$ The data from our study could not confirm these findings, since we did not find a statistically significant difference in survival between patients with $18 \mathrm{q} \mathrm{LOH}$ and stable tumors when treated with capecitabine adjuvant monotherapy both with univariate and with multivariate analyses. However, in models stratified according to clinicopathologic features, surprisingly, we observed a positive predictive impact of $18 \mathrm{q} \mathrm{LOH}$ on survival in patients with a distal localization of the tumors regardless of the tumor stage. We cannot give a definite explanation for these contradictory findings. However, all the studies (including ours) used different markers and criteria for assessing $18 \mathrm{q} \mathrm{LOH}$, which could confound the results and cause discrepant findings. Thus, it would be beneficial to develop a consensus panel of markers to assess 


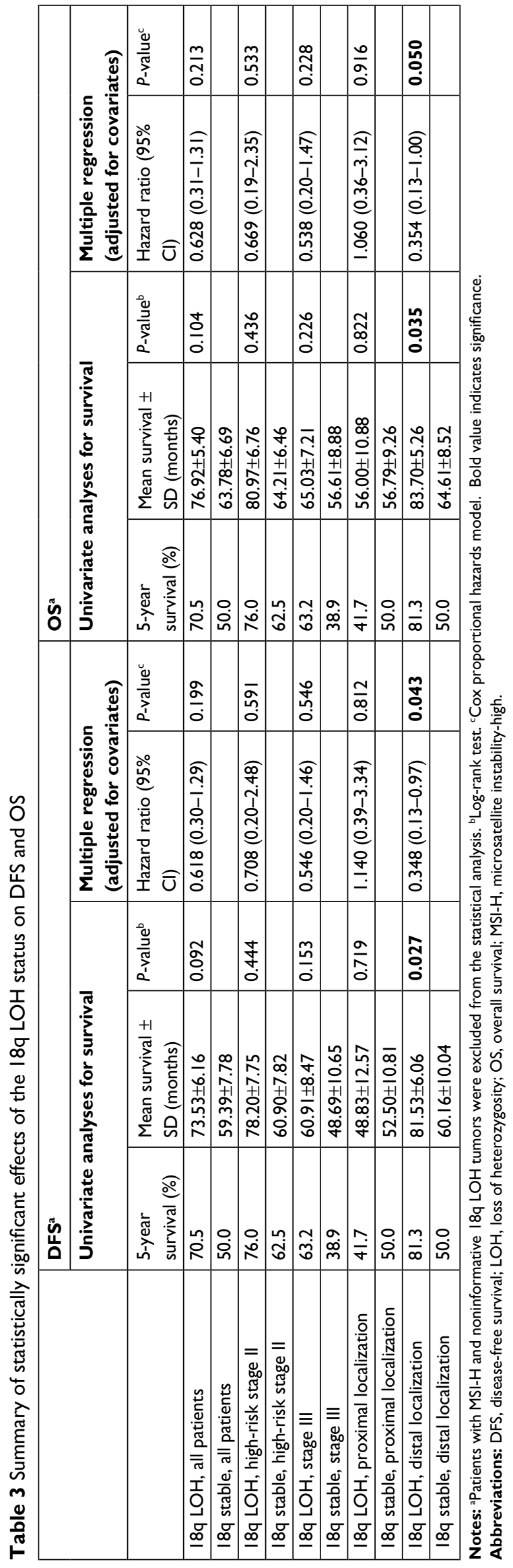

$18 \mathrm{q} \mathrm{LOH}$ in colon cancer in future trials. Also, the differing association might be partly due to the small sample size and thus further studies should be conducted in order to provide a definite conclusion. However, in our study, the survival rate of patients with $18 \mathrm{q} \mathrm{LOH}$, distal colon cancer treated with capecitabine as monotherapy in an adjuvant setting is very high (81.3\%) which should not be left unnoted. As a comparison, a previously published Phase III clinical trial (NO16968) evaluating Xeloda with oxaliplatin (XELOX) vs bolus 5-FU/ $\mathrm{LV}$ as adjuvant therapy in unselected stage III colon cancer patients reported $70.9 \%$ DFS in the XELOX arm. ${ }^{49}$ Further studies are needed, but if the survival rates of patients with $18 \mathrm{q} \mathrm{LOH}$, distal colon cancer treated with capecitabine as a monotherapy in an adjuvant setting are similar with those treated with multidrug regimens, then pretreatment testing for $18 \mathrm{q} \mathrm{LOH}$ might be beneficial in the selection of patients eligible for capecitabine monotherapy as first-line treatment.

The major mechanism of action of 5-FU and the prodrug capecitabine is the inhibition of TYMS through an active metabolite, fluorodeoxyuridylate, which forms a ternary complex with TYMS and 5,10-methylenetetrahydrofolate (5,10-MTHF). ${ }^{50}$ MTHFR catalyzes the irreversible conversion of 5,10-MTHF to 5-methyltetrahydrofolate and thus controls the intracellular levels of 5,10-MTHF ${ }^{50}$ Both TYMS and MTHFR variants have the potential to modify the efficacy of 5-FU-based treatment in CRC. VNTR polymorphism in the TYMS 5 -untranslated region has been associated with an altered TYMS expression and clinical response, with high expressing variants showing lower rates of response and toxicity. ${ }^{51-53}$ The common MTHFR polymorphism C677T is associated with depleted enzymatic activity which leads to alteration of intracellular folate distribution and enhanced 5-FU activity. ${ }^{53-55}$ The results from our study did not show a significant association of TYMS 5'VNTR and MTHFR C677T variants with DFS or OS in patients treated with capecitabine adjuvant monotherapy, leading to the conclusion that isolated variants in MTHFR or TYMS probably cannot be used to predict the effect or therapeutic failure.

\section{Conclusion}

Both MSI and 18q LOH markers have the potential to be utilized in the selection of colon cancer patients who will benefit from capecitabine adjuvant monotherapy. Patients with high-risk stage II and stage III colon cancer with proximal MSI-H tumors that arise from serrated adenomas and patients with distal 18q LOH, MSS colon cancer have better outcomes regardless of the stage of the disease and are potential candidates for capecitabine monotherapy. 
A

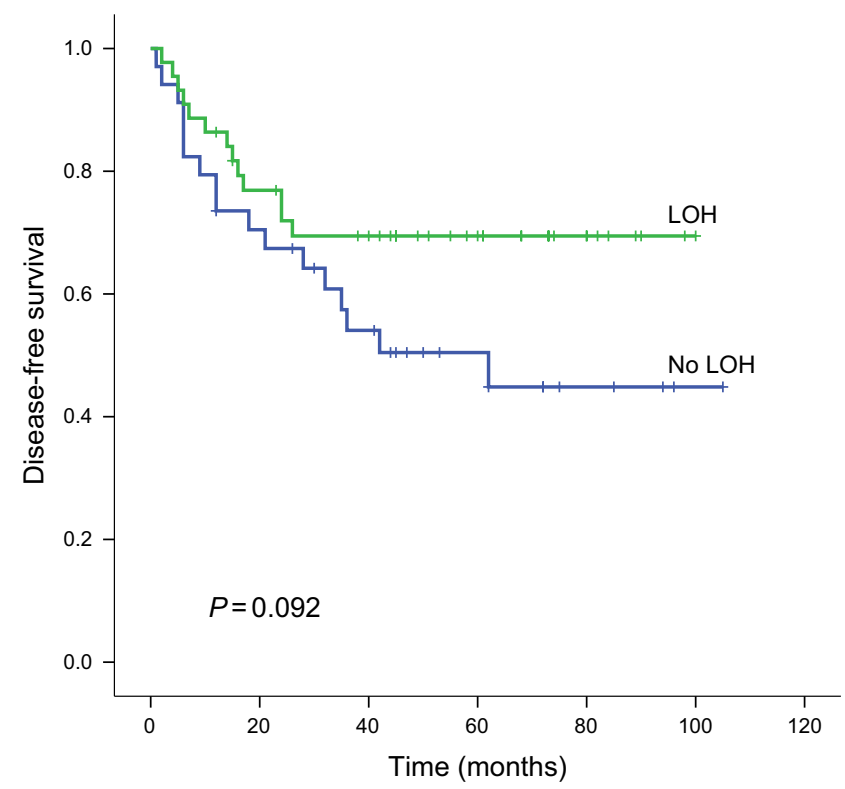

B

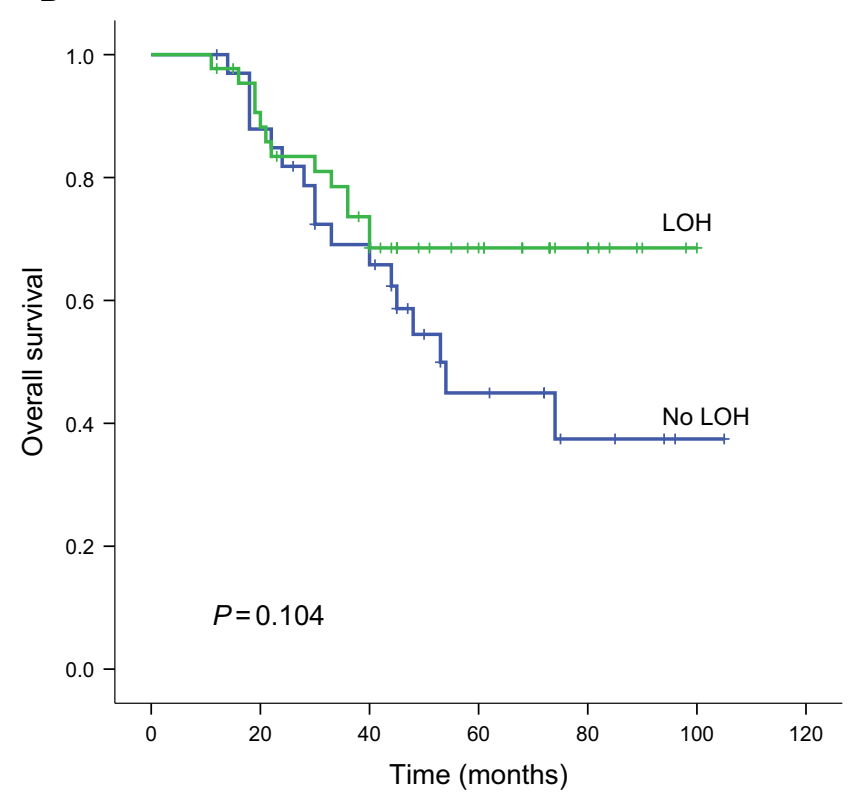

Figure 3 Kaplan-Meier estimates for disease-free survival (A) and overall survival (B) by the I8q LOH status in patients treated with capecitabine adjuvant monotherapy. Abbreviation: $\mathrm{LOH}$, loss of heterozygosity.

A

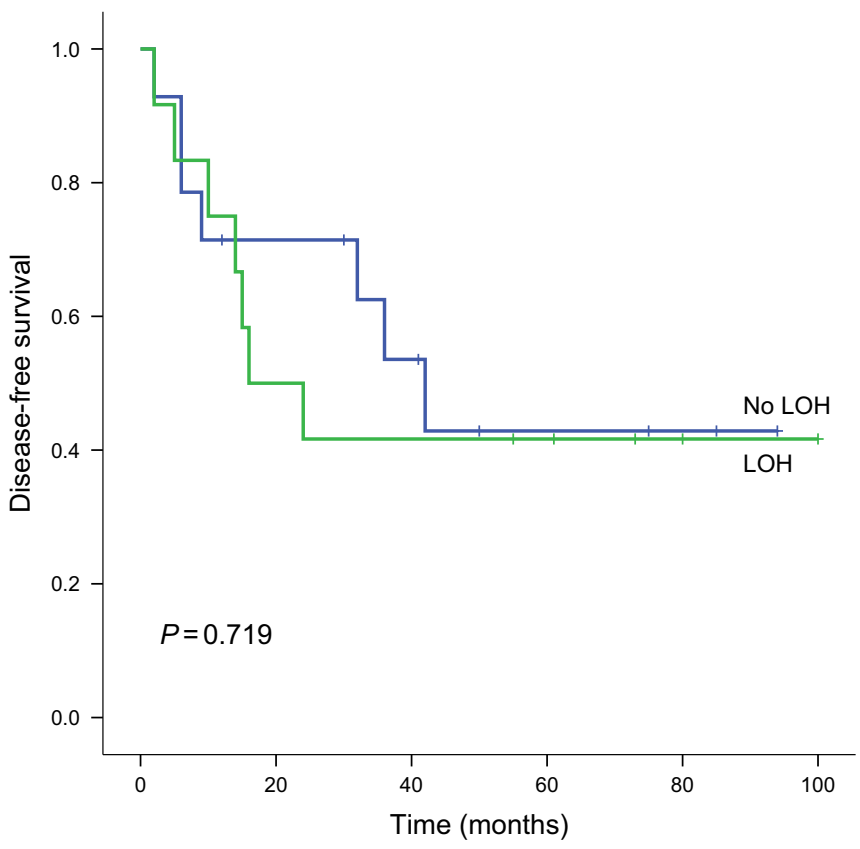

B

Distal localization

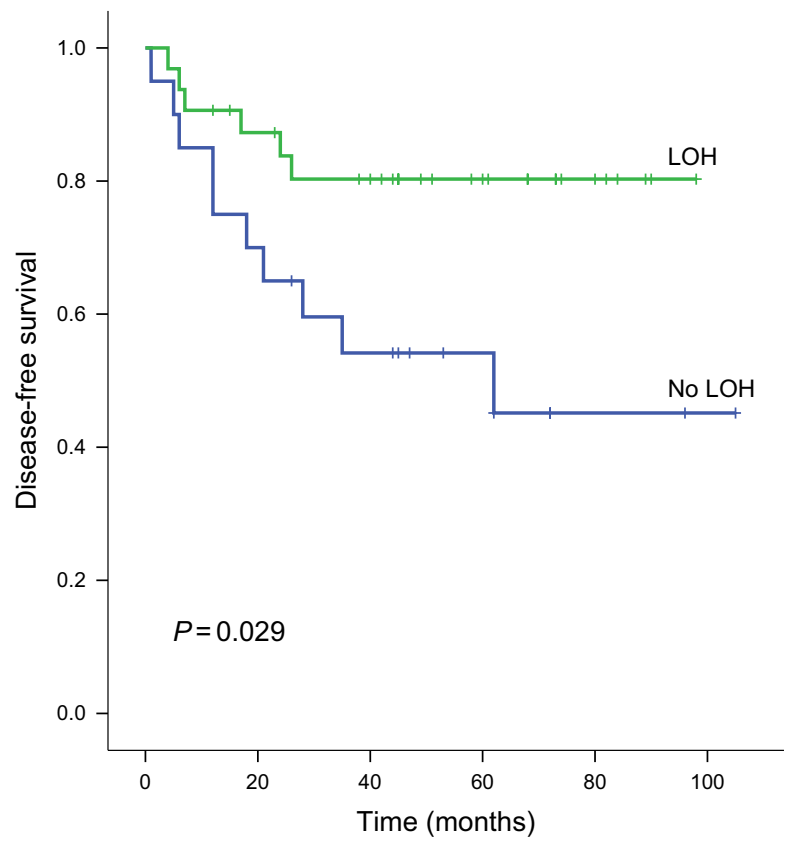

Figure 4 Kaplan-Meier curves of disease-free survival according to the $18 \mathrm{q} \mathrm{LOH}$ status of the tumor among patients with microsatellite stable colon cancer with localization proximal $(\mathbf{A})$ and distal $(\mathbf{B})$ to the splenic flexure.

Abbreviation: LOH, loss of heterozygosity.

\section{Acknowledgments}

This research work was supported by Hoffmann-La Roche, Representative Office Skopje, Macedonia (local open-label trial, ML20122) and a grant from the Ministry of Education and Science of the Republic of Macedonia (13-3584/1).

\section{Author contributions}

All authors made substantial contributions to conception and design, acquisition of data, or analysis and interpretation of data; took part in drafting the article or revising it critically for important intellectual content; gave final approval of the 
version to be published; and agree to be accountable for all aspects of the work.

\section{Disclosure}

The authors report no conflicts of interest in this work.

\section{References}

1. Ferlay J, Soerjomataram I, Dikshit R, et al. Cancer incidence and mortality worldwide: sources, methods and major patterns in GLOBOCAN 2012. Int J Cancer. 2015;136(5):E359-E386.

2. Ferlay J, Soerjomataram I, Ervik M. GLOBOCAN 2012 v1.0. Cancer incidence and mortality worldwide: IARC cancer base no 11 [webpage on the Internet]. Lyon, France: International Agency for Research on Cancer; 2013. Available from: http://globocan.iarc.fr. Accessed March 17, 2017.

3. Binefa G, Rodríguez-Moranta F, Teule A, Medina-Hayas M. Colorectal cancer: from prevention to personalized medicine. World J Gastroenterol. 2014;20(22):6786-6808.

4. Twelves C, Wong A, Nowacki MP, et al. Capecitabine as adjuvant treatment for stage III colon cancer. NEngl J Med. 2005;352(26):2696-2704.

5. Van Cutsem E, Hoff PM, Harper P, et al. Oral capecitabine vs intravenous 5-fluorouracil and leucovorin: integrated efficacy data and novel analyses from two large, randomised, phase III trials. $\mathrm{Br} J$ Cancer. 2004;90(6):1190-1197.

6. Cassidy J, Douillard JY, Twelves C, et al. Pharmacoeconomic analysis of adjuvant oral capecitabine vs intravenous 5-FU/LV in Dukes' $\mathrm{C}$ colon cancer: the X-ACT trial. Br J Cancer. 2006;94(8):1122-1129.

7. Eggington S, Tappenden P, Pandor A, et al. Cost-effectiveness of oxaliplatin and capecitabine in the adjuvant treatment of stage III colon cancer. Br J Cancer. 2006;95(9):1195-1201.

8. Johnston PG. Stage II colorectal cancer: to treat or not to treat. Oncologist. 2005;10(5):332-334.

9. Boland CR, Thibodeau SN, Hamilton SR, et al. A National Cancer Institute Workshop on Microsatellite Instability for cancer detection and familial predisposition: development of international criteria for the determination of microsatellite instability in colorectal cancer. Cancer Res. 1998;58(22):5248-5257.

10. Laiho P, Launonen $\mathrm{V}$, Lahermo $\mathrm{P}$, et al. Low-level microsatellite instability in most colorectal carcinomas. Cancer Res. 2002;62(4):1166-1170.

11. Samowitz WS, Curtin K, Ma KN, et al. Microsatellite instability in sporadic colon cancer is associated with an improved prognosis at the population level. Cancer Epidemiol Biomarkers Prev. 2001;10(9):917-923.

12. Wright CM, Dent OF, Barker M, et al. Prognostic significance of extensive microsatellite instability in sporadic clinicopathological stage $\mathrm{C}$ colorectal cancer. Br J Surg. 2000;87(9):1197-1202.

13. Khan SA, Morris M, Idrees K, et al. Colorectal cancer in the very young: a comparative study of tumor markers, pathology and survival in early onset and adult onset patients. J Pediatr Surg. 2016;51(11):1812-1817.

14. Kim CG, Ahn JB, Jung M, et al. Effects of microsatellite instability on recurrence patterns and outcomes in colorectal cancers. Br J Cancer. 2016;115(1):25-33.

15. Popat S, Hubner R, Houlston RS. Systematic review of microsatellite instability and colorectal cancer prognosis. J Clin Oncol. 2005;23(3):609-618.

16. NCC Network. NCCN clinical practice guidelines in oncology: colon cancer (version 2); 2016. Available from: https://www.nccn.org/professionals/physician_gls/f_guidelines.asp. Accessed January 15, 2017.

17. Benatti P, Gafà R, Barana D, et al. Microsatellite instability and colorectal cancer prognosis. Clin Cancer Res. 2005;11(23):8332-8340.

18. Jover R, Zapater P, Castells A, et al. The efficacy of adjuvant chemotherapy with 5-fluorouracil in colorectal cancer depends on the mismatch repair status. Eur J Cancer. 2009;45(3):365-373.
19. Guastadisegni C, Colafranceschi M, Ottini L, Dogliotti E. Microsatellite instability as a marker of prognosis and response to therapy: a metaanalysis of colorectal cancer survival data. Eur J Cancer. 2010;46(15): 2788-2798.

20. Ribic CM, Sargent DJ, Moore MJ, et al. Tumor microsatellite-instability status as a predictor of benefit from fluorouracil-based adjuvant chemotherapy for colon cancer. $N$ Engl J Med. 2003;349(3):247-257.

21. Sargent DJ, Marsoni S, Monges G, et al. Defective mismatch repair as a predictive marker for lack of efficacy of fluorouracil-based adjuvant therapy in colon cancer. J Clin Oncol. 2010;28(20):3219-3226.

22. Lamberti C, Lundin S, Bogdanow M, et al. Microsatellite instability did not predict individual survival of unselected patients with colorectal cancer. Int J Colorectal Dis. 2007;22(2):145-152.

23. Kim GP, Colangelo LH, Wieand HS, et al. Prognostic and predictive roles of high-degree microsatellite instability in colon cancer: a National Cancer Institute-National Surgical Adjuvant Breast and Bowel Project Collaborative Study. J Clin Oncol. 2007;25(7):767-772.

24. Des Guetz G, Uzzan B, Nicolas P, Schischmanoff O, Perret GY, Morere JF. Microsatellite instability does not predict the efficacy of chemotherapy in metastatic colorectal cancer. A systematic review and meta-analysis. Anticancer Res. 2009;29(5):1615-1620.

25. Webber EM, Kauffman TL, O'Connor E, Goddard KA. Systematic review of the predictive effect of MSI status in colorectal cancer patients undergoing 5FU-based chemotherapy. BMC Cancer. 2015;15:156.

26. Hemminki A, Mecklin JP, Järvinen H, Aaltonen LA, Joensuu H. Microsatellite instability is a favorable prognostic indicator in patients with colorectal cancer receiving chemotherapy. Gastroenterology. 2000;119(4):921-928.

27. Elsaleh H, Joseph D, Grieu F, Zeps N, Spry N, Iacopetta B. Association of tumour site and sex with survival benefit from adjuvant chemotherapy in colorectal cancer. Lancet. 2000;355(9217):1745-1750.

28. Hutchins G, Southward K, Handley K, et al. Value of mismatch repair, KRAS, and BRAF mutations in predicting recurrence and benefits from chemotherapy in colorectal cancer. J Clin Oncol. 2011;29(10): $1261-1270$

29. Klingbiel D, Saridaki Z, Roth AD, Bosman FT, Delorenzi M, Tejpar S. Prognosis of stage II and III colon cancer treated with adjuvant 5-fluorouracil or FOLFIRI in relation to microsatellite status: results of the PETACC-3 trial. Ann Oncol. 2015;26(1):126-132.

30. Tejpar S, Saridaki Z, Delorenzi M, Bosman F, Roth AD. Microsatellite instability, prognosis and drug sensitivity of stage II and III colorectal cancer: more complexity to the puzzle. J Natl Cancer Inst. 2011;103(11):841-844.

31. Sinicrope FA, Foster NR, Thibodeau SN, et al. DNA mismatch repair status and colon cancer recurrence and survival in clinical trials of 5-fluorouracil-based adjuvant therapy. $J$ Natl Cancer Inst. 2011;103(11):863-875.

32. Des Guetz G, Schischmanoff O, Nicolas P, Perret GY, Morere JF, Uzzan B. Does microsatellite instability predict the efficacy of adjuvant chemotherapy in colorectal cancer? A systematic review with meta-analysis. Eur J Cancer. 2009;45(10):1890-1896.

33. Park DY, Sakamoto H, Kirley SD, et al. The Cables gene on chromosome $18 \mathrm{q}$ is silenced by promoter hypermethylation and allelic loss in human colorectal cancer. Am J Pathol. 2007;171(5):1509-1519.

34. Alazzouzi H, Alhopuro P, Salovaara R, et al. SMAD4 as a prognostic marker in colorectal cancer. Clin Cancer Res. 2005;11(7):2606-2611.

35. Watanabe T, Wu TT, Catalano PJ, et al. Molecular predictors of survival after adjuvant chemotherapy for colon cancer. $N$ Engl $J$ Med. 2001;344(16):1196-1206

36. Jen J, Kim H, Piantadosi S, et al. Allelic loss of chromosome 18q and prognosis in colorectal cancer. $N$ Engl J Med. 1994;331(4): 213-221.

37. Ogunbiyi OA, Goodfellow PJ, Herfarth K, et al. Confirmation that chromosome $18 \mathrm{q}$ allelic loss in colon cancer is a prognostic indicator. J Clin Oncol. 1998;16(2):427-433. 
38. Jernvall P, Mäkinen MJ, Karttunen TJ, Mäkelä J, Vihko P. Loss of heterozygosity at $18 \mathrm{q} 21$ is indicative of recurrence and therefore poor prognosis in a subset of colorectal cancers. Br J Cancer. 1999;79(5-6): 903-908.

39. Sarli L, Bottarelli L, Bader G, et al. Association between recurrence of sporadic colorectal cancer, high level of microsatellite instability, and loss of heterozygosity at chromosome 18q. Dis Colon Rectum. 2004;47(9):1467-1482.

40. Martínez-López E, Abad A, Font A, et al. Allelic loss on chromosome $18 \mathrm{q}$ as a prognostic marker in stage II colorectal cancer. Gastroenterology. 1998;114(6):1180-1187.

41. Roth AD, Tejpar S, Delorenzi M, et al. Prognostic role of KRAS and BRAF in stage II and III resected colon cancer: results of the translational study on the PETACC-3, EORTC 40993, SAKK 60-00 trial J Clin Oncol. 2010;28(3):466-474.

42. Ogino S, Nosho K, Irahara N, et al. Prognostic significance and molecular associations of $18 \mathrm{q}$ loss of heterozygosity: a cohort study of microsatellite stable colorectal cancers. J Clin Oncol. 2009;27(27): 4591-4598.

43. Popat S, Zhao D, Chen Z, et al. Relationship between chromosome $18 \mathrm{q}$ status and colorectal cancer prognosis: a prospective, blinded analysis of 280 patients. Anticancer Res. 2007;27(1B):627-633.

44. Halling KC, French AJ, McDonnell SK, et al. Microsatellite instability and $8 \mathrm{p}$ allelic imbalance in stage B2 and C colorectal cancers. J Natl Cancer Inst. 1999;91(15):1295-1303.

45. Carethers JM, Hawn MT, Greenson JK, Hitchcock CL, Boland CR. Prognostic significance of allelic lost at chromosome 18q21 for stage II colorectal cancer. Gastroenterology. 1998;114(6):1188-1195.

46. Bertagnolli MM, Redston M, Compton CC, et al. Microsatellite instability and loss of heterozygosity at chromosomal location 18q: prospective evaluation of biomarkers for stages II and III colon cancer - a study of CALGB 9581 and 89803. J Clin Oncol. 2011;29(23):3153-3162.
47. Mouradov D, Domingo E, Gibbs P, et al. Survival in stage II/III colorectal cancer is independently predicted by chromosomal and microsatellite instability, but not by specific driver mutations. Am J Gastroenterol. 2013;108(11):1785-1793.

48. Barratt PL, Seymour MT, Stenning SP, et al. DNA markers predicting benefit from adjuvant fluorouracil in patients with colon cancer: a molecular study. Lancet. 2002;360(9343):1381-1391.

49. Haller DG, Tabernero J, Maroun J, et al. Capecitabine plus oxaliplatin compared with fluorouracil and folinic acid as adjuvant therapy for stage III colon cancer. J Clin Oncol. 2011;29(11):1465-1471.

50. Longley DB, Harkin DP, Johnston PG. 5-fluorouracil: mechanisms of action and clinical strategies. Nat Rev Cancer. 2003;3(5):330-338.

51. Pullarkat ST, Stoehlmacher J, Ghaderi V, et al. Thymidylate synthase gene polymorphism determines response and toxicity of 5-FU chemotherapy. Pharmacogenomics J. 2001;1(1):65-70.

52. Lecomte T, Ferraz JM, Zinzindohoué F, et al. Thymidylate synthase gene polymorphism predicts toxicity in colorectal cancer patients receiving 5-fluorouracil-based chemotherapy. Clin Cancer Res. 2004;10(17):5880-5888.

53. Jakobsen A, Nielsen JN, Gyldenkerne N, Lindeberg J. Thymidylate synthase and methylenetetrahydrofolate reductase gene polymorphism in normal tissue as predictors of fluorouracil sensitivity. J Clin Oncol. 2005;23(7):1365-1369.

54. Etienne MC, Formento JL, Chazal M, et al. Methylenetetrahydrofolate reductase gene polymorphisms and response to fluorouracil-based treatment in advanced colorectal cancer patients. Pharmacogenetics. 2004;14(12):785-792.

55. Cohen V, Panet-Raymond V, Sabbaghian N, Morin I, Batist G, Rozen R. Methylenetetrahydrofolate reductase polymorphism in advanced colorectal cancer: a novel genomic predictor of clinical response to fluoropyrimidine-based chemotherapy. Clin Cancer Res. 2003;9(5):1611-1615.
Pharmacogenomics and Personalized Medicine

\section{Publish your work in this journal}

Pharmacogenomics and Personalized Medicine is an international, peerreviewed, open access journal characterizing the influence of genotype on pharmacology leading to the development of personalized treatment programs and individualized drug selection for improved safety, efficacy and sustainability. This journal is indexed on the American Chemical

\section{Dovepress}

Society's Chemical Abstracts Service (CAS). The manuscript management system is completely online and includes a very quick and fair peer-review system, which is all easy to use. Visit http://www.dovepress. com/testimonials.php to read real quotes from published authors. 